\title{
FDTD Models for Complex Materials
}

\author{
Andrew D. Greenwood*
}

Air Force Research Laboratory, Directed Energy Directorate, Kirtland AFB, NM 87117-5776 USA

\begin{abstract}
Complex materials are of increasing interest in Finite-Difference Time-Domain modeling. For example, when the particle density becomes large, collisional fluid models of plasmas are an attractive alternative to particle in cell methods. Further, frequency dispersive meta-materials are of increasing interest. Thus, Finite-Difference Time-Domain (FDTD) models are derived for magnetized plasmas and for the Lorentz and Drude material models. Previous models of these types of materials make assumptions that may unnecessarily restrict the simulation time step. By considering the solution of the differential equations on the interval of a time step, these assumptions are avoided. Studies show that the resulting magnetized plasma model is numerically stable when the FDTD Courant condition and the Nyquist sampling theorem for the plasma and cyclotron frequencies are obeyed. Waves propagating in the modeled plasma exhibit the correct dispersion relations. Studies also show the Lorentz and Drude material models to be stable up to the FDTD Courant limit and to exhibit the correct dispersion relations.
\end{abstract}

Keywords: FDTD, plasma, lorentz material, drude material.

\section{INTRODUCTION}

The finite-difference time-domain FDTD method [1] successfully models a variety of electromagnetic phenomena in a variety of materials. One phenomenon of interest is the propagation of electromagnetic waves in a magnetized plasma, which behaves as a gyro-tropic medium. While other authors also consider magnetized plasmas [2-4], the model considered here (1) models the plasma currents at the Yee FDTD electric field locations, (2) avoids a matrix inversion, and (3) does not restrict the time step to a value less than the Nyquist sampling theorem for the cyclotron frequency. Also of interest are frequency dispersive meta-materials, which are modeled as Lorentz or Drude materials. Similar to magnetized plasmas, other authors also consider Lorentz and Drude materials $[5,6]$. However, the formulation considered here avoids assumptions that unnecessarily may limit the FDTD time step.

\section{FORMULATION}

\subsection{Magnetized Plasma}

The magnetized plasma formulation is based on the Lorentz force law for charged particles, given by

$$
\frac{\partial \mathbf{v}}{\partial t}+v \mathbf{v}=\frac{q}{m}(\mathbf{E}+\mathbf{v} \times \mathbf{B})
$$

where $\mathbf{v}$ is the average velocity of the particle distribution, $v$ is the collision frequency, $q$ is charge, $m$ is mass, $\mathbf{E}$ is the electric field, and $\mathbf{B}$ is the magnetic field. Note that plasmas of interest here consist of positive ions that are assumed infinitely massive and electrons; thus $q$ and $m$ are the electron charge and mass, respectively. The magnetic

*Address correspondence to this author at the Air Force Research Laboratory, Directed Energy Directorate, Kirtland AFB, NM 87117-5776 USA; Tel: 505-846-6642; Fax: 505-846-0566;

E-mail: agreenwood@ieee.org field consists of a static component $\mathbf{B}_{0}$ and an electrodynamic component $\mathbf{B}_{d}$. Thus, Eq. (1) can be written

$$
\frac{\partial \mathbf{v}}{\partial t}+v \mathbf{v}=\frac{q}{m}\left(\mathbf{E}+\mathbf{v} \times \mathbf{B}_{d}+\mathbf{v} \times \mathbf{B}_{0}\right)
$$

from the solution of Maxwell's equations, $E \approx c B_{d}$ where $c$ is the speed of light, and for problems of interest, the average particle velocity $v<<c$; thus, $E \gg v B_{d}$, and the second term on the right hand side of Eq. (2) is neglected, resulting in

$$
\frac{\partial \mathbf{v}}{\partial t}+v \mathbf{v}=\frac{q}{m}\left(\mathbf{E}+\mathbf{v} \times \mathbf{B}_{0}\right)
$$

Eq. (3) is multiplied by the plasma number density $N$ times the charge $q$, and the definitions for current density $(\mathbf{J}=N q \mathbf{v})$, plasma frequency $\left(\omega_{p}^{2}=N q^{2} /\left(m \varepsilon_{0}\right)\right)$, and cyclotron frequency $\left(\Omega_{c}=q \mathbf{B}_{0} / m\right)$ are applied, giving

$$
\frac{\partial \mathbf{J}}{\partial t}+v \mathbf{J}=\varepsilon_{0} \omega_{p}^{2} \mathbf{E}-\Omega_{c} \times \mathbf{J}
$$

note that in Eqs. (1)-(4), the fluid advection term is neglected. This limits the use of the model to cases where the fluid flow is negligible during the time of the simulation.

Eq. (4) is really three coupled differential equations. The equations can be decoupled and a tensor permittivity derived [7], but this leads to a third order differential equation that is more difficult to discretize. The coupled equations on the staggered Yee FDTD grid can also lead to a matrix inversion for the solution, which nearly all previous works seek to avoid due to its high numerical cost. In [3], a matrix inversion is avoided by placing all components of $\mathbf{J}$ in the center of a cell, while in [4], it is avoided by solving Eq. (4), then dealing with the staggered grid. However, the solution 
in [4] restricts the simulation time step $\left(\Delta_{t}\right)$ such that $\omega_{c} \Delta_{t}<<1\left(\omega_{c}=\left|\boldsymbol{\Omega}_{c}\right|\right)$.

To remove the time step restriction imposed by the solution in [4], Eq. (4) is solved on the interval $\left[(n-0.5) \Delta_{t},(n+0.5) \Delta_{t}\right]$ where $n$ is a positive integer by assuming a constant value of $\mathbf{E}=\mathbf{E}^{n}=\mathbf{E}\left(t=n \Delta_{t}\right)$. The solution takes the form

$$
\begin{aligned}
& \mathbf{J}=\mathbf{C}_{0}+\mathbf{C}_{1} e^{-v\left[t-(n-0.5) \Delta_{t}\right]}+\mathbf{C}_{2} e^{-v\left[t-(n-0.5) \Delta_{t}\right]} \cos \left\{\omega_{c}\left[t-(n-0.5) \Delta_{t}\right]\right\} \\
& +\mathbf{C}_{3} e^{-v\left[t-(n-0.5) \Delta_{t}\right]} \sin \left\{\omega_{c}\left[t-(n-0.5) \Delta_{t}\right]\right\}
\end{aligned}
$$

substitution of Eq. (5) into Eq. (4) and applying the initial condition $\mathbf{J}\left[t=(n-0.5) \Delta_{t}\right]=\mathbf{J}^{n-0.5}$ allows $\mathbf{C}_{0}-\mathbf{C}_{3}$ to be evaluated, giving

$$
\begin{aligned}
& \mathbf{J}^{n+0.5}=\left\{\begin{array}{l}
\mathbf{J}^{n-0.5} \cos \left(\omega_{c} \Delta_{t}\right)+\left[\hat{\Omega}_{c} \cdot \mathbf{J}^{n-0.5}\right] \hat{\Omega}_{c}\left[1-\cos \left(\omega_{c} \Delta_{t}\right)\right] \\
-\hat{\Omega}_{c} \times \mathbf{J}^{n-0.5} \sin \left(\omega_{c} \Delta_{t}\right)
\end{array}\right\} e^{-v \Delta_{t}} \\
& +\frac{\varepsilon_{0} \omega_{p}^{2}}{\omega_{c}^{2}+v^{2}}\left\{v\left[1-\cos \left(\omega_{c} \Delta_{t}\right) e^{-v \Delta_{t}}\right]+\omega_{c} \sin \left(\omega_{c} \Delta_{t}\right) e^{-v \Delta_{t}}\right\} \mathbf{E}^{n} \\
& -\frac{\varepsilon_{0} \omega_{p}^{2}}{\omega_{c}^{2}+v^{2}}\left\{\omega_{c}\left[1-\cos \left(\omega_{c} \Delta_{t}\right) e^{-v \Delta_{t}}\right]-v \sin \left(\omega_{c} \Delta_{t}\right) e^{-v \Delta_{t}}\right\}\left\{\hat{\Omega}_{c} \times \mathbf{E}^{n}\right\} \\
& +\frac{\varepsilon_{0} \omega_{p}^{2}}{\omega_{c}^{2}+v^{2}}\left\{\begin{array}{l}
\left.\frac{1-e^{-v \Delta_{t}}}{v} \omega_{c}^{2}-v\left[1-\cos \left(\omega_{c} \Delta_{t}\right)\right] e^{-v \Delta_{t}}\right\}\left\{\hat{\Omega}_{c} \cdot \mathbf{E}^{n}\right\} \hat{\Omega}_{c} \\
-\omega_{c} \sin \left(\omega_{c} \Delta_{t}\right) e^{-v \Delta_{t}}
\end{array}\right.
\end{aligned}
$$

where $\hat{\Omega}_{c}$ is the unit vector in the direction of $\boldsymbol{\Omega}_{c}$. Note that applying L'Hospital's rule reduces Eq. (6) to well defined limits when $\omega_{c}=0$ and/or $v=0$. When components of $\mathbf{E}$ and $\mathbf{J}$ from other spatial locations on the staggered grid are needed in Eq. (6), averages of the four surrounding components (second order accurate) are used. This requires some storing and tracking of which $\mathbf{J}$ components are already updated, but does not require a matrix inversion, which is numerically intractable for large computations. Note that in the staggered FDTD grid [1] there are four surrounding components to average in either 2-D or 3-D simulations. Further note that the update equations in [4] can be found by using 1 and 2 term series approximations for $\sin \left(\omega_{c} \Delta_{t}\right)$ and $\cos \left(\omega_{c} \Delta_{t}\right)$, which require that $\omega_{c} \Delta_{t} \ll<1$ to ensure $\sin \left(\omega_{c} \Delta_{t}\right) \approx \omega_{c} \Delta_{t}$ and $\cos \left(\omega_{c} \Delta_{t}\right) \approx 1-\omega_{c}^{2} \Delta_{t}^{2} / 2$. Using the exact value of $\sin \left(\omega_{c} \Delta_{t}\right)$ and $\cos \left(\omega_{c} \Delta_{t}\right)$ in the current derivation removes the $\omega_{c} \Delta_{t}<<1$ restriction.

The standard stability analysis by substituting solutions of the form

$G^{n} e^{i\left(l k_{x} \Delta_{x}+J k_{y} \Delta_{y}+K k_{z} \Delta_{z}\right)}$

is undertaken to analyze the stability of Eq. (6) together with the standard Yee FDTD discretizations of Ampere's Law and Faraday's Law. The result is a $9 \times 9$ matrix whose determinant is a ninth degree polynomial in $G$; the roots of that polynomial must be less than or equal to one in magnitude for stability. Unfortunately, even with modern computer techniques, expressions found for the roots are too complex to gain any insight. However, the behavior of the roots can be studied numerically for specific scenarios. For example, specific values of $\boldsymbol{\Omega}_{c}, \omega_{p}$, and the spatial discretization parameters $\left(\Delta_{x}, \Delta_{y}, \Delta_{z}\right)$ can be substituted into the equations, and then the roots can be found for different time steps and wave numbers on the grid. Such studies find that the system is stable up to the usual FDTD Courant limit

$$
\Delta_{t} \leq \frac{1}{c}\left(\sqrt{\frac{1}{\Delta_{x}^{2}}+\frac{1}{\Delta_{y}^{2}}+\frac{1}{\Delta_{z}^{2}}}\right)^{-1}
$$

( $c$ is the speed of light) when $\omega_{p} \Delta_{t}<\pi$ and $\omega_{c} \Delta_{t}<\pi$. Cases where $\omega_{p} \Delta_{t}>\pi$ or $\omega_{c} \Delta_{t}>\pi$ violate the Nyquist sampling theorem and lead to aliasing of $\omega_{p}$ and/or $\omega_{c}$. Studies show that this can but does not always lead to numerical instability. However, it is sure to lead to poor numerical accuracy even if it does not lead to numerical instability.

\subsection{Meta-Materials}

The Lorentz and Drude material models can be used to match the frequency response of many meta-materials of interest. For the Lorentz material model ( $e^{-i \omega t}$ time dependence suppressed),

$\varepsilon(\omega)=\varepsilon_{0}\left(\varepsilon_{r}+\frac{\omega_{e p}^{2}}{\omega_{e 0}^{2}-i \gamma_{e} \omega-\omega^{2}}\right)$

and

$\mu(\omega)=\mu_{0}\left(\mu_{r}+\frac{\omega_{m p}^{2}}{\omega_{m 0}^{2}-i \gamma_{m} \omega-\omega^{2}}\right)$

where $\omega_{e p}$ and $\omega_{m p}$ are the plasma frequencies, $\omega_{e 0}$ and $\omega_{m 0}$ are the resonance frequencies, and $\gamma_{e}$ and $\gamma_{m}$ are the absorption parameters [5]. The Drude model is a special case of the Lorentz model when $\omega_{e 0}=0$ and/or $\omega_{m 0}=0$. Eqs. (9) and (10) are sometimes written [6]

$\varepsilon(\omega)=\varepsilon_{\infty}-\frac{\omega_{e 0}^{2}\left(\varepsilon_{s}-\varepsilon_{\infty}\right)}{\omega^{2}+2 i \omega \delta_{e}-\omega_{e 0}^{2}}$

and

$\mu(\omega)=\mu_{\infty}-\frac{\omega_{m 0}^{2}\left(\mu_{s}-\mu_{\infty}\right)}{\omega^{2}+2 i \omega \delta_{m}-\omega_{m 0}^{2}}$

The two forms can be shown to be equivalent, and the form in Eqs. (9) and (10) is preferred because it more conveniently reduces to the Drude model. Some metamaterials exhibit anisotropic properties with diagonal permittivity and permeability tensors. Modeling such is a simple extension to the model presented here (simply specify different parameters for the $x x, y y$, and $z z$ components of the tensor and apply the correct parameters to the component 
field updates in the code), but this capability is not yet coded and tested.

Because Eqs. (9) and (10) are identical in form, the electric field derivation only is presented. The magnetic field derivation is identical. To find the electric field FDTD update, Eq. (9) is substituted into $\mathbf{D}=\varepsilon \mathbf{E}$ and transformed into a time-domain differential equation

$$
\frac{\partial^{2} \mathbf{D}}{\partial t^{2}}+\gamma_{e} \frac{\partial \mathbf{D}}{\partial t}+\omega_{e 0}^{2} \mathbf{D}=\varepsilon_{0} \varepsilon_{r}\left(\frac{\partial^{2} \mathbf{E}}{\partial t^{2}}+\gamma_{e} \frac{\partial \mathbf{E}}{\partial t}+\left(\omega_{e 0}^{2}+\omega_{e p}^{2} / \varepsilon_{r}\right) \mathbf{E}\right)
$$

similar to the magnetized plasma derivation above, to avoid any unnecessary time step restriction, the solution for $\mathbf{E}$ as a function of $\mathbf{D}$ is computed on the interval $t=\left[(n-1) \Delta_{t},(n+1) \Delta_{t}\right]$ where $\Delta_{t}$ is the simulation time step and $n$ is a positive integer. Note that $\mathbf{D}$ is advanced in the simulation using Ampere's law. On $t=\left[(n-1) \Delta_{t},(n+1) \Delta_{t}\right]$,

$\mathbf{D} \approx 0.5 \mathbf{D}_{2 a} \tau^{2}+\mathbf{D}_{1 a} \tau+\mathbf{D}^{n}(14)$

where $\tau=t-n \Delta_{t}, \mathbf{D}^{n}=\mathbf{D}\left(t=n \Delta_{t}\right)$,

$\mathbf{D}_{2 a}=\left(\mathbf{D}^{n+1}-2 \mathbf{D}^{n}+\mathbf{D}^{n-1}\right) / \Delta_{t}^{2}$,

and

$\mathbf{D}_{1 a}=\left(\mathbf{D}^{n+1}-\mathbf{D}^{n-1}\right) /\left(2 \Delta_{t}\right)$. Thus, the form of the solution for $\mathbf{E}$ is

$\mathbf{E}=e^{-\gamma} e^{\tau / 2}\left(\mathbf{C}_{1} \cos \alpha \tau+\mathbf{C}_{2} \sin \alpha \tau\right)+\mathbf{C}_{3} \tau^{2}+\mathbf{C}_{4} \tau+\mathbf{C}_{5}$

where $\alpha=\sqrt{\omega_{e 0}^{2}+\omega_{e p}^{2} / \varepsilon_{r}-\gamma_{e}^{2} / 4}$ and $\mathbf{C}_{1}-\mathbf{C}_{5}$ are constants to be determined. These constants are determined by substituting Eqs. (14) and (15) into Eq. (13) and using the initial conditions $\mathbf{E}\left(t=(n-1) \Delta_{t}\right)=\mathbf{E}^{n-1} \quad$ and $\mathbf{E}\left(t=n \Delta_{t}\right)=\mathbf{E}^{n}$, resulting in the following update expression for $\mathbf{E}^{n+1}$ :

$\mathbf{E}^{n+1}=2 e^{-\gamma_{e} \Delta_{t} / 2} \cos \alpha \Delta_{t} \mathbf{E}^{n}-e^{-\gamma_{e} \Delta_{t}} \mathbf{E}^{n-1}$

$+\frac{1}{\varepsilon_{0} \varepsilon_{r}}\left\{\frac{\omega_{e 0}^{2}}{\omega_{e 0}^{2}+\omega_{e p}^{2} / \varepsilon_{r}}\left[\mathbf{D}^{n+1}-2 e^{-\gamma_{e} \Delta_{t} / 2} \cos \alpha \Delta_{t} \mathbf{D}^{n}+e^{-\gamma_{e} \Delta_{t}} \mathbf{D}^{n-1}\right]\right.$

$+\frac{\gamma_{e} \omega_{e p}^{2} / \varepsilon_{r}}{\left(\omega_{e 0}^{2}+\omega_{e p}^{2} / \varepsilon_{r}\right)^{2}}\left[\begin{array}{l}\mathbf{D}_{1 a}\left(1-2 e^{-\gamma_{e} \Delta_{t} / 2} \cos \alpha \Delta_{t}+e^{-\gamma_{e} \Delta_{t}}\right) \\ +\mathbf{D}_{2 a} \Delta_{t}\left(1-e^{-\gamma_{e} \Delta_{t}}\right)\end{array}\right]$

$\left.+\frac{\omega_{e p}^{2}}{\varepsilon_{r}} \frac{\omega_{e 0}^{2}+\omega_{e p}^{2} / \varepsilon_{r}-\gamma_{e}^{2}}{\left(\omega_{e 0}^{2}+\omega_{e p}^{2} / \varepsilon_{r}\right)^{3}} \mathbf{D}_{2 a}\left[1-2 e^{-\gamma_{e} \Delta_{t} t^{2}} \cos \alpha \Delta_{t}+e^{-\gamma_{e} \Delta_{t}}\right]\right\}$

Note that Taylor series approximations for the cosine and exponential terms can be used in the update expression above. However, doing so can impose time step restrictions when $\omega_{e p}, \omega_{e 0}$, or $\gamma_{e}$ are large. This update expression is derived to avoid such restrictions. Note, however, that to satisfy the Nyquist sampling theorem, $\alpha \Delta_{t}=\Delta_{t} \sqrt{\omega_{e 0}^{2}+\omega_{e p}^{2} / \varepsilon_{r}-\gamma_{e}^{2} / 4}$

must be less than $\pi$. The performance penalty for computing the cosine and exponential terms on modern computers is not large. Further, because these terms are not likely to change during the simulation, the performance penalty can be reduced by precomputation.

Based on the above derivation, the procedure for advancing the fields in a Lorentz or Drude material is

compute $\mathbf{D}^{n+1}=f\left(\mathbf{H}^{n+0.5}, \mathbf{D}^{n}\right)$ using the discretized Ampere's law,

compute $\mathbf{E}^{n+1}=f\left(\mathbf{D}^{n+1}, \mathbf{D}^{n}, \mathbf{D}^{n-1}, \mathbf{E}^{n}, \mathbf{E}^{n-1}\right)$ using Eq. ((16)),

compute $\mathbf{B}^{n+1.5}=f\left(\mathbf{E}^{n+1}, \mathbf{B}^{n+0.5}\right)$ using the discretized Faraday's law,

compute $\mathbf{H}^{n+1.5}=f\left(\mathbf{B}^{n+1.5}, \mathbf{B}^{n+0.5}, \mathbf{B}^{n-0.5}, \mathbf{H}^{n+0.5}, \mathbf{H}^{n-0.5}\right)$ using the magnetic analog to Eq. (16),

increment $\mathrm{n}$ and repeat.

Similar to the magnetized plasma, it is difficult to gain insight from a symbolic stability analysis. However, specific cases can be proved stable, and experience shows that the numerical stability limit is not less than the free space FDTD Courant condition. Note that "backwards wave" materials where $\mu, \varepsilon<0$ for some range of frequencies can be successfully modeled with the Lorentz and Drude materials.

\section{NUMERICAL RESULTS}

The magnetized plasma model is implemented and tested in the Improved Concurrent Electromagnetic Particle-In-Cell (ICEPIC) code. For the magnetized plasma simulations discussed below, the cubic cells have a side length of 1.07 $\mathrm{mm}$ (40 cells per wavelength at $7 \mathrm{GHz}$ ), and the time step is 2.04 ps $(99 \%$ of the FDTD Courant limit). The computational domain is surrounded by perfectly matched layer (PML) absorbing boundary conditions. Waves in a magnetized plasma propagating parallel to the magnetic field decompose into right-hand circular polarized and left-hand circular polarized waves that propagate at different speeds, leading to the phenomenon known as Faraday rotation. Waves propagating parallel to the magnetic field are simulated in a plasma with $v=0, f_{p}=1 \mathrm{GHz}$ $\left(\omega_{p}=6.28 \times 10^{9} / \mathrm{s}\right)$, and $f_{c}=5 \mathrm{GHz}\left(\omega_{c}=3.14 \times 10^{10} / \mathrm{s}\right)$ at frequencies from $0.1 \mathrm{GHz}$ to $7.0 \mathrm{GHz}$, and the wave number is computed at each frequency. The resulting dispersion diagrams are compared with analytically computed diagrams in Fig. (1).

The percent error in the computed wave number is also plotted in Fig. (1). In most cases, the error is less than 1 percent. The error appears to increase when the wave number is small; however, the error increase is due to the finite resolution of the Fourier transform used to compute the wave number from the simulation results rather than the result of the model breaking down $\left(\Delta_{k} / k\right.$ increases when $k$ is small; $\Delta_{k}$ is the resolution of the Fourier transform and $k$ 
(a) Right-Hand Circular Polarization

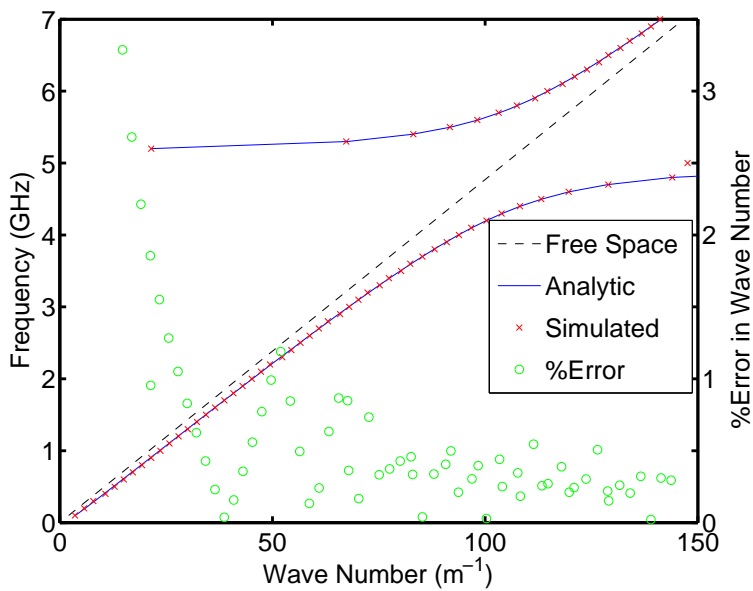

(b) Left-Hand Circular Polarization

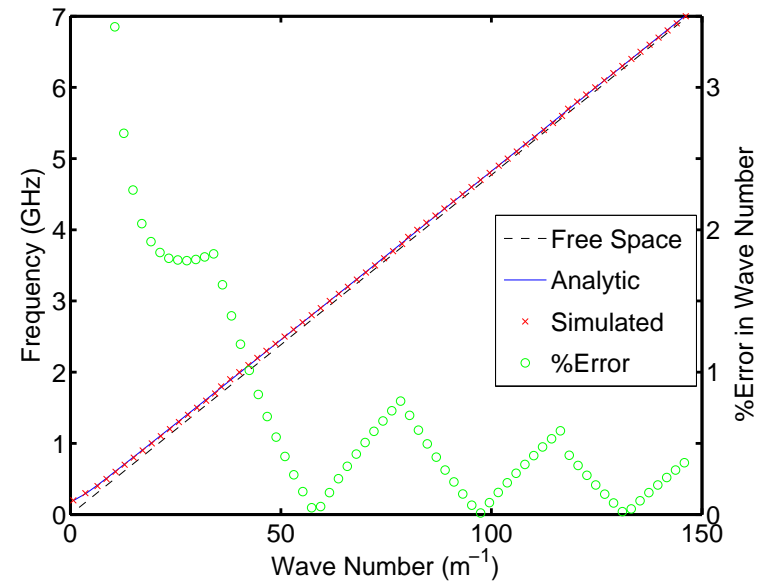

Fig. (1). (a, b) Dispersion diagrams of waves propagating parallel to the static magnetic field in a plasma with $v=0, f_{p}=1 \mathrm{GHz}$ $\left(\omega_{p}=6.28 \times 10^{9} / \mathrm{s}\right)$, and $f_{c}=5 \mathrm{GHz}\left(\omega_{c}=3.14 \times 10^{10} / \mathrm{s}\right)$.

is the wave number). Waves propagating perpendicular to the static magnetic field are linearly polarized, and the dispersion diagram is a function of whether the electric field is polarized parallel or perpendicular to the static magnetic field.

Waves propagating perpendicular to the magnetic field are simulated in the same plasma as above, and the resulting dispersion diagrams are shown in Fig. (2).

Similar to Fig. (1), the percent error in the computed wave number is also shown in Fig. (2). Also similar to Fig. (1), the error increase when the wave number is small is due to the finite resolution of the Fourier transform used to compute the wave number rather than due to a breakdown if the model. The reflection coefficient from a $10 \mathrm{~cm}$ thick plasma layer is also computed and compared with analytic results. With the possible exception of very low frequency, the results shown in Figs. $(\mathbf{3}, \mathbf{4})$ show excellent agreement.
The percent error in the reflection coefficient is not shown because the reflection nulls dominate the error rather than phenomena related to the computational algorithm; however, away from a reflection null, the error is generally less than 2 percent. Other tests of the algorithm (results not shown) include computing the dispersion diagram for a nonmagnetized plasma (result looks similar to Fig. (2a) as it should) and running a simulation for several hundred thousand time steps to ensure long time numerical stability (no instability observed).

The Lorentz/Drude material model is also implemented and tested in ICEPIC. For the simulations below, the size of the FDTD cells is $0.2 \mathrm{~mm}$ (100 cells per wavelength at 15 $\mathrm{GHz})$, and the time step is 0.381 ps $(99 \%$ of the FDTD Courant limit). The computational domain is surrounded by PML absorbing boundary conditions. The test material has (a) Parallel Polarization

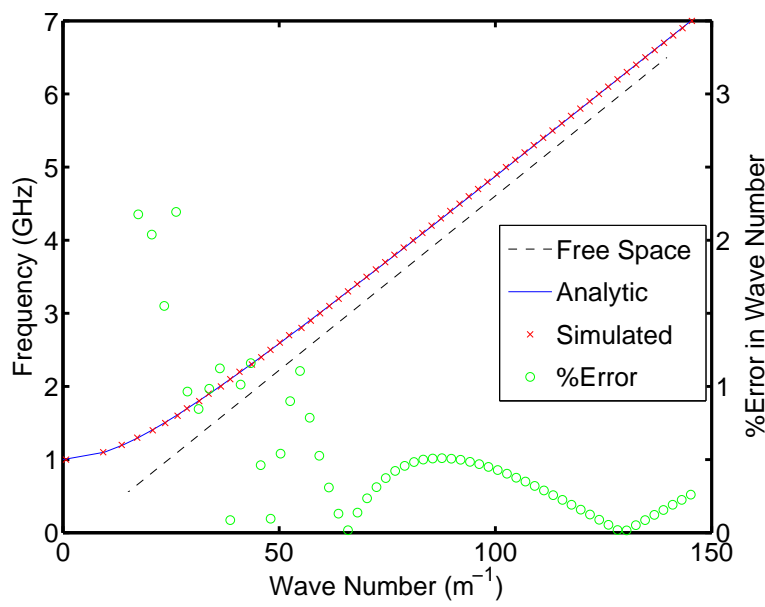

(b) Perpendicular Polarization

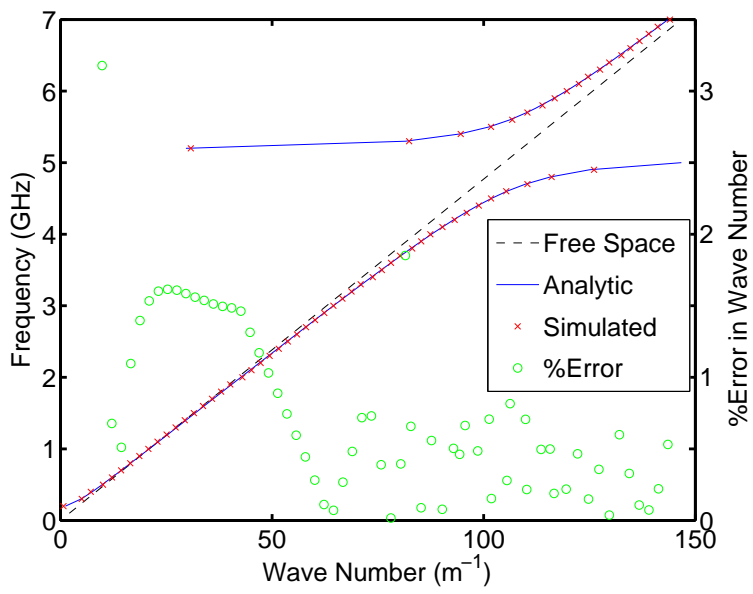

Fig. (2). (a, b) Dispersion diagrams of waves propagating perpendicular to the static magnetic field in a plasma with $v=0, f_{p}=1 \mathrm{GHz}$ $\left(\omega_{p}=6.28 \times 10^{9} / \mathrm{s}\right)$, and $f_{c}=5 \mathrm{GHz}\left(\omega_{c}=3.14 \times 10^{10} / \mathrm{s}\right)$. 
(a) Right-Hand Circular Polarization

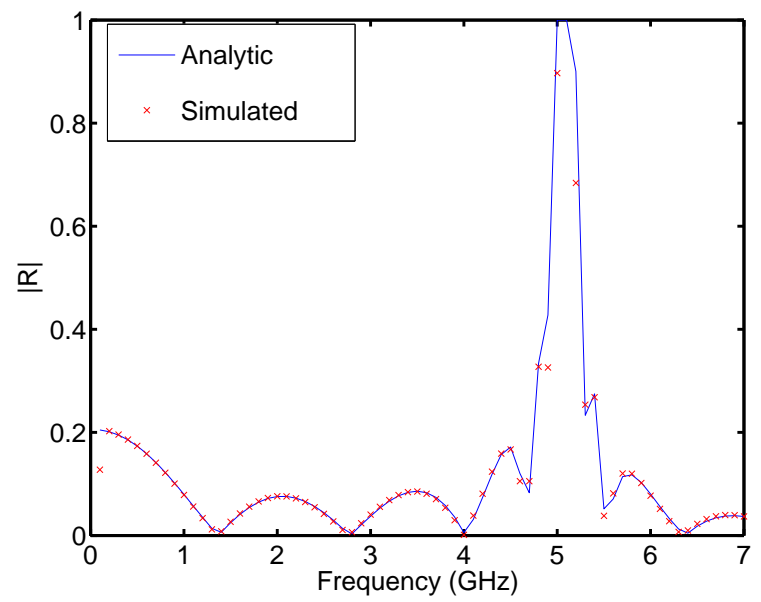

(b) Left-Hand Circular Polarization

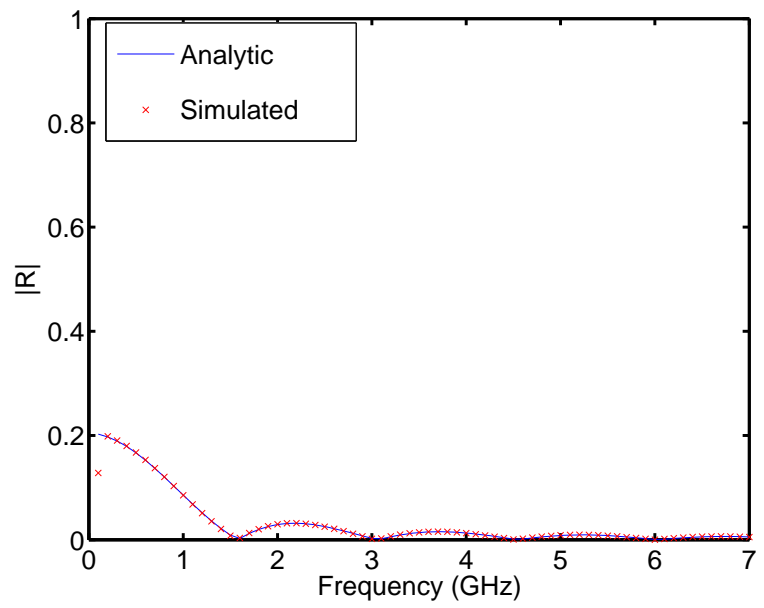

Fig. (3). (a, b) Reflection coefficient from a $10 \mathrm{~cm}$ thick plasma layer with propagation parallel to the static magnetic field. The plasma layer is characterized by $v=0, f_{p}=1 \mathrm{GHz}\left(\omega_{p}=6.28 \times 10^{9} / \mathrm{s}\right)$, and $f_{c}=5 \mathrm{GHz}\left(\omega_{c}=3.14 \times 10^{10} / \mathrm{s}\right)$.

(a) Parallel Polarization

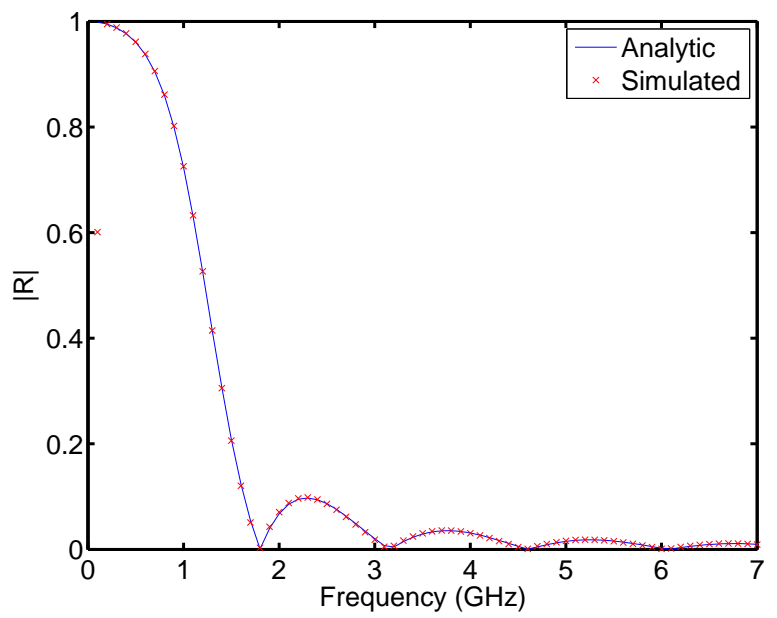

(b) Perpendicular Polarization

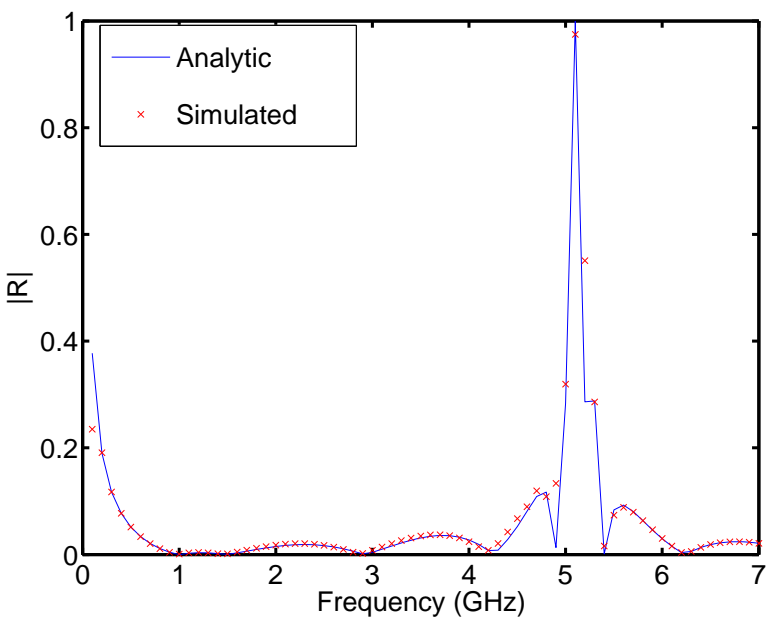

Fig. (4). (a, b) Reflection coefficient from a $10 \mathrm{~cm}$ thick plasma layer with propagation perpendicular to the static magnetic field. The plasma layer is characterized by $v=0, f_{p}=1 \mathrm{GHz}\left(\omega_{p}=6.28 \times 10^{9} / \mathrm{s}\right)$, and $f_{c}=5 \mathrm{GHz}\left(\omega_{c}=3.14 \times 10^{10} / \mathrm{s}\right)$.

$\mu_{r}=\varepsilon_{r}=1, \quad \omega_{e p}=\omega_{m p}=30 \pi \sqrt{2} \mathrm{GHz}, \quad \omega_{e 0}=\omega_{m 0}=2 \pi 5$ $\mathrm{MHz}$, and $\gamma_{e}=\gamma_{m}=2 \pi 5 \mathrm{MHz}$. This material is characterized by $\mu / \mu_{0}=\varepsilon / \varepsilon_{0} \approx-1$ at $f=15 \mathrm{GHz}$. The material is highly dispersive, as shown in Fig. (5) where it is also seen that the simulated results agree very closely with the analytic results. The percent error is less than 1 percent until the wave number becomes small; as discussed above, when the wave number is small, the resolution of the Fourier transform used to compute the wave number from the simulation results limits the accuracy rather than a breakdown in the algorithm. Note in Fig. (5) that (1) the wave number decreases with increasing frequency, in contrast to the characteristics of free space or simple dielectric materials, and (2) the parameters of the simulated material are such that the imaginary part of the wave number is small and thus not shown. Another characteristic of this material is very slow wave propagation. Fig. (6) shows the analytic and simulated group velocities in the meta material, which again are in agreement one another (less than 2 percent error), and are significantly slower than the free space speed of light $(c)$. The reflection coefficient from a transition from free space to this meta-material is small; thus, a plot is uninteresting. A plot of the transmission coefficient through a $10 \mathrm{~cm}$ thick material layer (Fig. 7) shows that the simulation reflects increasing transmission with frequency, indicating that the loss becomes more important at lower frequency. Note that the percent error in Fig. (7) is below 0.5 percent except at low frequency, where it remains below 2 percent. 


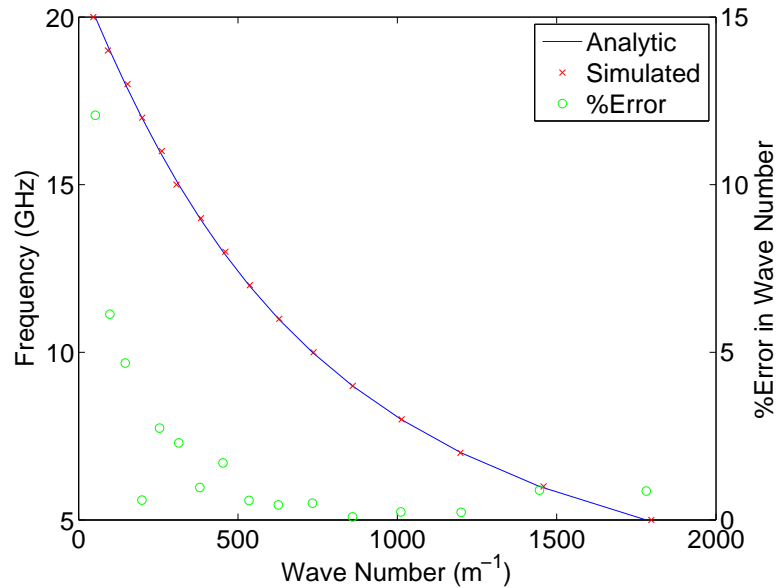

Fig. (5). Dispersion diagram of a meta-material with $\mu_{r}=\varepsilon_{r}=1$, $\omega_{e p}=\omega_{m p}=30 \pi \sqrt{2} \quad \mathrm{GHz}, \quad \omega_{e 0}=\omega_{m 0}=2 \pi 5 \quad \mathrm{MHz}, \quad$ and $\gamma_{e}=\gamma_{m}=2 \pi 5 \mathrm{MHz}$

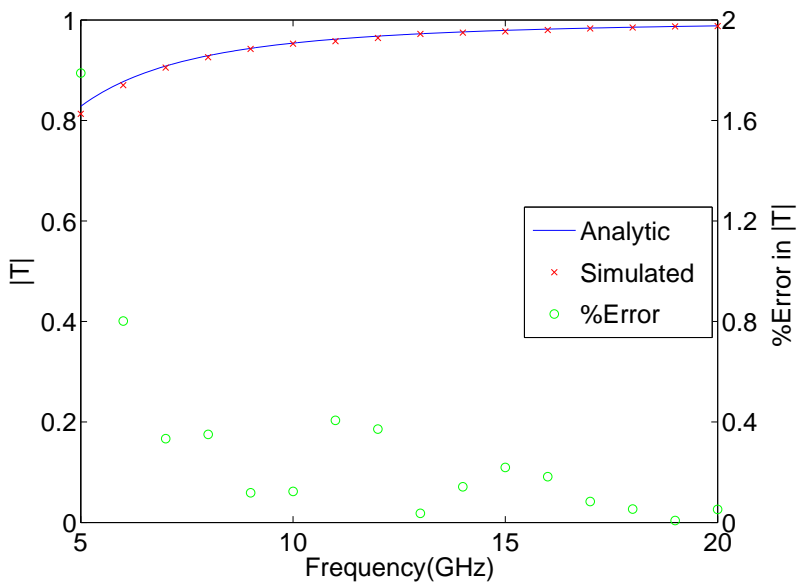

Fig. (7). Transmission through a $10 \mathrm{~cm}$ thick layer of meta-material with $\mu_{r}=\varepsilon_{r}=1, \quad \omega_{e p}=\omega_{m p}=30 \pi \sqrt{2} \mathrm{GHz}, \quad \omega_{e 0}=\omega_{m 0}=2 \pi 5$ $\mathrm{MHz}$, and $\gamma_{e}=\gamma_{m}=2 \pi 5 \mathrm{MHz}$.

\section{CONCLUSION}

FDTD models are derived for a magnetized plasma and Lorentz/Drude materials. The magnetized plasma model (1) models the plasma currents at the Yee FDTD electric field locations, (2) avoids a matrix inversion, and (3) does not restrict the time step to a value less than the Nyquist sampling theorem for the cyclotron frequency. The

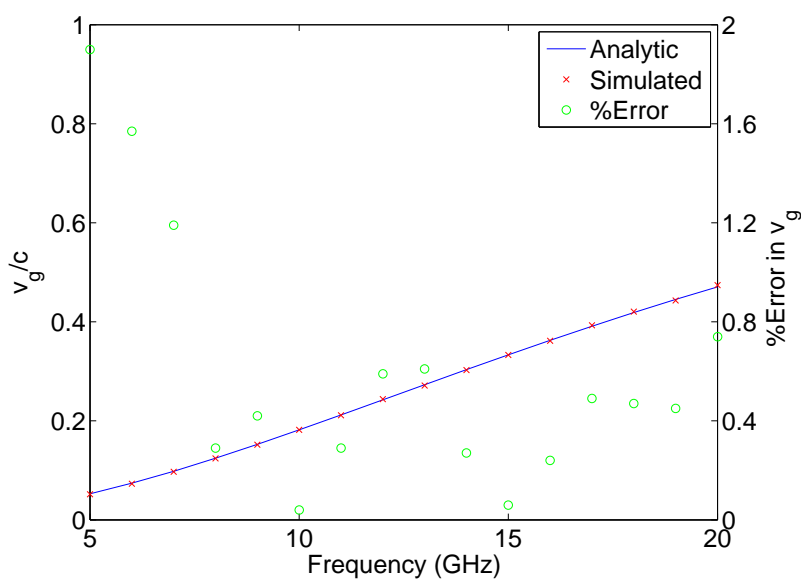

Fig. (6). Wave propagation speed (group velocity, $v_{g}$ ) in a metamaterial with $\mu_{r}=\varepsilon_{r}=1, \quad \omega_{e p}=\omega_{m p}=30 \pi \sqrt{2} \quad \mathrm{GHz}$, $\omega_{e 0}=\omega_{m 0}=2 \pi 5 \mathrm{MHz}$, and $\gamma_{e}=\gamma_{m}=2 \pi 5 \mathrm{MHz}$.

Lorentz/Drude material model also avoids restricting the time step to a value less than the Nyquist sampling theorem for the plasma or resonance frequencies in the model. Numerical tests show that the models captures the relevant physics of electromagnetic waves in a magnetized plasmas and meta-materials modeled as Lorentz materials.

\section{REFERENCES}

[1] Yee KS. Numerical solution of initial boundary value problems involving Maxwell's equations in isotropic media. IEEE Trans Antennas Propagat 1966; 14: 302-07.

[2] Hunsberger F, Luebbers RJ, Kunz KS. Finite difference time domain analysis of gyrotropic media-I: magnetized plasma. IEEE Trans Antennas Propagat 1992; 40: 1489-95.

[3] Lee JH, Kalluri DK. Three-dimensional FDTD simulation of EM wave transformation in a dynamic inhomogeneous magnetized plasma. IEEE Trans Antennas Propagat 1999; 47: 1146-51.

[4] Xu L, Yuan N. FDTD formulations for scattering from 3-D anisotropic magnetized plasma objects. IEEE Antennas Wireless Propagat Lett 2006; 5: 335-8.

[5] Feise MW, Schneider JB, Bevelacqua PJ. Finite-Difference and Pseudospectral Time-Domain Methods Applied to BackwardsWave Metamaterials. IEEE Trans Antennas Propagat 2004; 52: 2955-62.

[6] Joseph RM, Hagness SC, Taflove A. Direct time integration of Maxwells equations in linear dispersive media with absorption for scattering and propagation of femtosecond electromagnetic pulses. Opt Lett 1991; 16: 1412-4.

[7] Kong JA. Electromagnetic Wave Theory. New York: John Wiley \& Sons, Inc. 1990.

This is an open access article licensed under the terms of the Creative Commons Attribution Non-Commercial License (http://creativecommons.org/licenses/by$\mathrm{nc} / 3.0 /$ ) which permits unrestricted, non-commercial use, distribution and reproduction in any medium, provided the work is properly cited. 